\title{
ON
}

\section{A NEW METHOD OF OPERATING}

\section{IOR \\ I M P E R M E A B L E U R E T H R A.}

BY

JAMES SYME, F.R.S.E.

PROFESSOR OF CLINICAL SURGERY IN THE UNIVRRSITY OF EDINBURGH.

Received Jan. 3d.-Read Feb. 24th, 1857.

IN the communication on the remedy of stricture by external incision, which upon a former occasion was submitted to this society, I endeavoured to show that the nature of stricture was inconsistent with impermeability, and that whenever the urine could pass through the urethra, an instrument might be introduced into the bladder. It was far from my intention to allege that any surgeon could accomplish this in every case, at once and with ease. Many patient trials may be requisite before success is attained, but, with time and care, my belief was, and is, that no degree or form of contraction can be truly considered as impermeable. Still less, if possible, was it my intention to deny that, in consequence of wounds or sloughing, the urethra might become completely obstructed beyond the fistulous opening, in regard both to the exit of urine and to the introduction of instruments. Such cases, though certainly rare, unquestionably do occasionally occur, and when presenting themselves, have hitherto proved very troublesome subjects of treatment.

There being no passage for the introduction of instruments, it has been deemed necessary to cut upon the point of a $\mathbf{X} \mathbf{x}$. 
catheter passed down to the seat of obstruction, so as to clear the way for its conveyance into the bladder, where it may remain until the textures concerned are sufficiently condensed to prevent extravasation of urine. Nothing can be more simple in theory, or apparently more easy of execution, than this procedure; but any one who has undertaken it, or witnessed its performance, must be prepared to admit that there are few operations in surgery so embarrassing and uncertain. The thickened and indurated state of the perinæum, together with the distortion of form apt to result from the wound or sloughing that caused the fistulous opening, render it extremely difficult, or rather, indeed, almost impossible, to cut exactly in the proper course of the urethra, while any deviation from it may produce the most serious consequences. Thus, if the knife strays in a lateral direction, it is pretty sure to occasion hæmorrhage; and, as ligatures are of no use in such circumstances, there must then be a resort to pressure, which, by impeding the escape of urine, will expose the patient to its injurious or fatal infiltration. If again the knife from cutting upon the end instead of the point of the catheter, as it is very apt to do, should not make a direct passage through the seat of obstruction, but leave a sort of projection between the two free parts of the canal,-although its immediate effect may prove less disastrous, the result can hardly be satisfactory in regard to either the completeness or permanency of recovery, since there will always be a hitch at the part, requiring instruments to be carefully guided past it by depressing the point, impeding the flow of urine, and increasing the tendency to contraction.

By one of those curious coincidences so frequently observed in surgical practice, two cases of obliterated urethra lately came under my care at the same time. One of these was that of William Scott, æt. 44, from Dunblane, admitted into the Royal Infirmary on the 21st of last October. $\mathrm{He}$ stated that on the 12th of August, in getting out of bed during the night, he slipped his foot and fell astride upon 
the back of a chair, so as to bruise the perinæum. Next morning, being unable to pass his water, he sent for a surgeon, who introduced a catheter and emptied the bladder. During the three following days he voided his urine with ease; but on the fourth began to suffer pain in micturition. Swelling in the scrotum and perinæum then took place; a slough the size of his hand separated from the former, and all his urine issued through an opening in the latter. After being reduced to a state of extreme weakness, he had in some degree regained general health; but never passed a drop of urine through the urethra. On examination, it was found impossible to pass any instrument through the seat of obstruction.

The second case was that of a seaman recommended to my care by Dr. Boyd, of St. John's, New Brunswick, who sent the following account of his case :

"Peter Scott, seaman, æt. 28, was admitted into the Provincial Marine Hospital, at St. John's, New Brunswick, on Thursday, 10th July, 1856, at 10 o'clock a.m.

"When going on board his ship last night about 12 o'clock, the night being very dark, he walked over the end of a quay, and fell astride a projecting log, twenty feet below the surface, and thence to the ground, whereby he received a very severe contusion on the perinæum, which, together with the penis and scrotum, were perfectly black from extravasated blood. I bled him, and applied cold lotion to the parts after placing him comfortably in bed.

" 6 p.m. Made very careful and repeated attempts to pass a catheter into the bladder. The instrument was passed with the greatest ease, and suddenly stopped after its whole length had been introduced, but no urine followed; there seemed to be a false passage. As he was not suffering inconvenience from desire to pass water, I considered it prudent not to persist. There was no bleeding from the urethra.

"11th. -9 a.m. Again attempted to pass a catheter, but with the same result. As the bladder was distended, and 
there was much desire to pass urine, I considered the case a very perilous one, and requested the advice of others. Upon consultation it was agreed upon to make an incision into the perinæum with the view of finding the urethra, and passing an instrument in that way into the bladder. Upon cutting into the perinæum much blood was found effused, and the coagulum being removed it was discovered that the urethra had been completely destroyed by the contusion, neither the bulb of the urethra, nor the prostate gland could be satisfactorily felt; there seemed to be a sort of sac which had been filled with blood. The os pubis was fractured near the symphisis. So extensive was the destruction of the parts anterior to the prostate gland that it was impossible to discover an orifice, and to relieve the bladder in that way : in order, therefore, to give him a chance, it was deemed necessary to tap the bladder above the pubis with a trocar, by which nearly two pints of urine were taken off. A gum catheter was then introduced through the canula, and the canula was withdrawn.

" 9 p.m. Complains of much pain in the back, has not the power of retaining stools, although he feels them passing. Pulse 98. Skin and tongue moist; has some thirst. Ordered lemonade.

"12th.-9 a.m. Has not had any sleep during the night; has passed a pint of urine by the tube; wound looks well; tumefaction of penis and scrotum much less, and the colour not so dark. Pulse 100. Belly soft; no pain upon pressure; slight pain shooting through the bladder occasionally; no headache; fell asleep in a few minutes after he had been made comfortable. Ordered cool drinks and quietness.

" $8 \frac{1}{2}$ p.m. Slept but a very short time; feels easy ; passed about three gills of urine by the catheter. Pulse 90, and full ; no pain on pressure; blood oozing from the wound. Penis and scrotum less swelled and discoloured; no general disturbance of the system.

Pergat.

"13th.-9 a.m. Bowels not open since operation; has 
had some sleep during the night. Pulse 90. Tongue white; urine high coloured, with deposit when cold.

\section{Olei Ricini, క̌ss.}

“ 8 p.m. No unfavorable symptoms. Pulse 98. Oil operated once slightly.

" 14th.-9 a.m. Complains of pains in his legs and back. Pulse 96. Tongue looks better. Bowels not open again.

Habeat Hydrargyri submuriatis, gr. v, statim.

" 9 p.m. Bowels have been moved four or five times; has no power over the sphincter ani. Dressed the wound with pledget of lint.

" 15 th to 29th.- Visited daily at the usual hours. No change worth recording from day to day. Urine continues to flow through the tube. Replaced Catheter No. 6, having withdrawn the one first introduced.

"August 6th.-Withdrew the catheter, as urine occasionally passes through the wound in the perinæum.

"9th. - Since the tube was withdrawn the urine has passed more freely through the wound, and he has the power of retaining it for two or three hours.

" 14th.-Wound above pubis quite healed : general health very good.

"October 8th.-It is unnecessary to detail the case further. The above abstract is given at the request of Scott, who has been discharged this day to proceed to sea.

$$
\begin{aligned}
& \text { (Signed) “ J. Boyd, M.D., \&c., } \\
& \text { “ Phys. and Surg. Prov. Mar. Hosp." }
\end{aligned}
$$

In considering how these cases were to be remedied, I felt great reluctance to adopt the usual operation, for the reasons which have been mentioned, and thought of another plan that promised to be more satisfactory, both in the performance and in the result. This was to introduce into the bladder through the fistulous opening-which, if necessary, might be dilated-a staff like that used in lithotomy, but with the groove on its concave instead of its convex 
side; then to insinuate through the urethra, so far as possible, the guide director employed for dividing strictures by external incision; and while the staff, confided to an assistant, was supported by a finger of the operator on the perinæum or in the rectum, to push the director onwards in the direction it ought to take if the canal were free, so as to pass through the obstructing texture, enter the groove, and proceed into the bladder. The state of matters being then similar to that of a stricture requiring division, after the director has been passed through it, there would be no difficulty in placing a knife in the groove and cutting outwards, so as to divide completely, in the exact line of the urethra, all the thickened substance concerned, and afford free admission to a full-sized catheter, which may be allowed to remain for two or three days to prevent any risk of extravasation. When it is necessary thus to reform completely a portion of the urethra, it would be unreasonable to expect a result equally speedy and satisfactory as when the natural canal merely requires to be enlarged. But I venture to hope that the plan of operating which has now been described may facilitate the procedure, and lessen the risk of bad consequences. 